International Journal of Pure and Applied Mathematics

Volume 85 No. 2 2013, 367-382

ISSN: 1311-8080 (printed version); ISSN: 1314-3395 (on-line version)

url: http://www.ijpam.eu

doi: http://dx.doi.org/10.12732/ijpam.v85i2.13

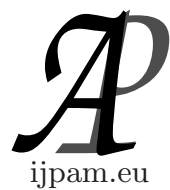

\title{
A GENERALIZED NASH EQUILIBRIUM PROBLEM FOR CENTRALIZED DYNAMIC SPECTRUM LEASING
}

\author{
Laura Prati \\ Department of Information Engineering \\ University of Florence \\ 3, Via S. Marta, I-50139 Florence, ITALY
}

\begin{abstract}
The dramatic increase of wireless devices in telecommunications is underlining the inefficiency of the traditional spectrum allocation system. A novel scheme for resource allocation is the Dynamic Spectrum Sharing (DSS), where primary users, the exclusive owners of the spectrum rights, allow the secondary ones to share their licensed frequency band.

In this paper, a time-continuous model is presented for Centralized Dynamic Spectrum Leasing (C-DSL). This kind of model involves the active participation of both the primary and secondary users in order to share the same frequency band.

I treat the problem of secondary power allocation like a non-cooperative Generalized Nash Equilibrium Problem (GNEP) where the primary communication system is willing to allow the secondary users to share its spectrum band and consequently adjusts the maximum tolerable interference in order to obtain the greatest gain, while the secondary users play the game by choosing the most convenient powers to be allocated, in order to obtain a good quality signal and avoid an excessive interference.

I give mathematical conditions, easy to be verified locally at the secondary receivers, that ensure the existence of a Generalized Nash Equilibrium for the game.
\end{abstract}

Received: January 7, 2013

(c) 2013 Academic Publications, Ltd. url: www.acadpubl.eu 
AMS Subject Classification: 90B18, 91A28, 91A40, 91A80

Key Words: game theory, generalized Nash equilibrium problem, cognitive radio, power allocation, resource allocation

\section{Introduction}

Recently, the problem of radio spectrum scarcity has been approached (see [1], [2]) and the inefficiency of the traditional static system for frequency band allocation has been shown. The scarcity of the radio spectrum is mainly due to the under-utilization of several spectrum bands dedicated to licensed users which are the exclusive owners of the spectrum rights (see [1], [3], [4], [5], [6]).

In the Introduction of [7] it is summarized how the problem of spectrum allocation has been faced in the course of time, even resorting to lotteries and auctions (the quoted paper indeed proposes a novel method for auctioning the radio spectrum). Moreover, innovative artificial techniques and new paradigms in radio communication protocols have been proposed to improve the use of a fixed band, see [8] and [9].

Nevertheless, another point of view is possible to improve the utilization of the radio spectrum: instead of transmitting signals on a formerly fixed frequency band, a radio transmitter might transmit its signal in a spectrum hole, leading to a dynamic and flexible condition.

A Cognitive Radio system, see [10] and [11], could be the answer for spectrum sensing: it is able to find spectrum holes by sensing the radio spectrum in the local neighborhood of the Cognitive Radio receivers in an unsupervised manner, see [12], [13] and [14].

In the so called Dynamic Spectrum Access (DSA), primary users, the owners of the spectrum rights in a certain frequency band, select a cap on the total interference the are willing to tolerate from the secondary ones, see [14], and the secondary users are expected to share the same band only when primary users are not transmitting; each primary user is a sort of spectator, in the sense that it does not play an active role. Primary users just do not tolerate an excessive interference: the smaller the secondary interference is, the bigger the satisfaction for primary users is. On the contrary, in the Dynamic Spectrum Leasing (DSL) the primary users have an active role in managing the interference they are going to tolerate, and they have an incentive, like monetary rewards, leasing payments, to allow the secondary users to share their band. While primary users manage their interference cap by varying it, if it is considered to be gainful, the secondary users manage their transmission powers, in order to obtain 
a satisfying signal quality without causing harmful interference. In this paper a Centralized Dynamic Spectrum Leasing (C-DSL) model is studied. It means that the multiple primary players participate in the spectrum leasing process under centralized control.

Game Theory is an appropriate tool to analyse the problem of power allocation and interference management chosen by users coexisting and competing in the same frequency band. Indeed, in a Game-theoretic model, the radio devices are players aiming to optimize their level of satisfaction by means of the choice of the interference cap, if primary users, or of their own transmitting power, if secondary users. Moreover, Game Theory is appropriate because of its distributed approach: decisions by secondary users are not taken in a centralized way, but every secondary player regulates its own power basing on locally available information, such as the total received interference.

Many papers have been proposed in the literature for secondary users coexistence and DSA models, see [15] for an overview. A Game-theoretic approach to the power control problem can be found in [16], [17], [18], [19] and [20], where utility functions to be maximized represent service preferences for the secondary users.

A major work is [21] where, in a secondary telecommunication system, two different models have been presented: a model with net utility for secondary users and another one with linear pricing added to the net utility. The net utility of [21] is the same that I have used in the present work, but I have chosen a different pricing, not linear, following [22] and [23], which also introduced the presence of a primary user in a DSA context. Actually, in [23] the discretization of time let the authors have a simplification in the mathematical treatment: indeed, by assuming that time is discrete, the pricing, instant after instant, reduces to a simpler linear function exactly as in [21].

The total utility for secondary users I have chosen is therefore the one proposed in [23], but with the introduction of multiple primary users and overall defining it in a continuous-time scenario. Moreover, the Game I propose is in a C-DSL context, letting the primary users play an active role and make their own decisions, see [24], [25] for DSL and [26] for C-DSL. The mathematical treatment is dedicated to the proof of existence of a Generalized Nash Equilibrium, see [27] and [28], in a Generalized Nash Equilibrium Problem (also named Coupled Constraint Equilibrium Problem) where the strategy sets of the mobiles are not independent of each other.

The paper is organized as follows: In Section 2 an overview on Generalized Nash Equilibrium Problems is given, in Section 3 the model is described, in Section 4 the Game is treated and in Section 5 I remark some conclusions. 


\section{Basics of Generalized Nash Equilibrium Problems (GNEPs)}

In this Section, I report the main definitions, nomenclature and results of the Generalized Nash Equilibrium Problems (GNEPs), formally introduced in [27] and [28] under the name of Abstract Economy. This Section is not intended to be a complete treatment of the topic. For an exhaustive survey on GNEP see for instance [29]. The GNEP consists of $N \geq 2$ players each controlling the variable $a_{i} \in A_{i}$ with $i \in \mathcal{N}=\{1,2, \ldots, N\}$. The sets $A_{i}$ are the strategy sets of the players and depend on the variables controlled by the other players.

Definition 1. We denote by $\boldsymbol{a}=\left(a_{1}, \ldots, a_{N}\right) \in A=A_{1} \ldots \times A_{N}$ the action vector formed by all the decision variables.

Remark on Notation. The action vector $\boldsymbol{a}=\left(a_{1}, \ldots, a_{N}\right) \in A$ is often represented by the shortened expression $\boldsymbol{a}=\left(a_{i}, \boldsymbol{a}_{-i}\right)$. This is to emphasize the choice $a_{i}$ of the $i$-th player with respect to the choices of the other $N-1$ players, represented by the index $-i$. Similarly, the set $A$ is often represented with the following notation: $A=A_{i} \times A_{-i}$.

Definition 2. The set $A=A_{1} \ldots \times A_{N}$ is the action space or strategy space. What makes a GNEP different from a traditional Game (or Nash Equilibrium Problem, NEP) is that the strategy set of each player depends on the variables of the other $N-1$ players, namely $A_{i}=A_{i}\left(\boldsymbol{a}_{-i}\right)$, for every $i \in \mathcal{N}$. The set $A_{i}\left(\boldsymbol{a}_{-i}\right)$ is called the feasible set of player $i$.

Each player aims to maximize its own utility function (or objective function) $u_{i}: A \rightarrow \mathbb{R}$, which gives an estimation of the players' preferences regarding the action vectors in $A$ : the $i$-th player prefers strategy $\boldsymbol{a} \in A$ with respect to strategy $\boldsymbol{a}^{\prime} \in A$ if and only if $u_{i}(\boldsymbol{a}) \geq u_{i}\left(\boldsymbol{a}^{\prime}\right)$ (large preference). Therefore we can define a GNEP:

Definition 3. A GNEP is the problem of finding a feasible action vector $\boldsymbol{a}^{*}$ with $a_{i}^{*} \in A_{i}\left(\boldsymbol{a}_{-i}^{*}\right)$ for every $i \in \mathcal{N}$ that solves the problem

$$
\underset{a_{i}}{\operatorname{maximize}} u_{i}\left(a_{i}, \boldsymbol{a}_{-i}\right) \quad \text { subject to } a_{i} \in A_{i}\left(\boldsymbol{a}_{-i}\right) \quad \text { for all } i \in \mathcal{N} \text {. }
$$

The vector $\boldsymbol{a}^{*} \in A\left(\boldsymbol{a}^{*}\right)$ where $A(\boldsymbol{a}):=\prod_{i=1}^{N} A_{i}\left(\boldsymbol{a}_{-i}\right)$ such that

$$
u_{i}\left(a_{i}^{*}, \boldsymbol{a}_{-i}^{*}\right) \geq u_{i}\left(a_{i}, \boldsymbol{a}_{-i}^{*}\right) \text { for every } a_{i} \in A_{i}\left(\boldsymbol{a}_{-i}^{*}\right) \text {, for all } i \in \mathcal{N}
$$

is called a Generalized Nash Equilibrium (GNE).

If the feasible sets $A_{i}$ do not depend on $\boldsymbol{a}_{-i}$, the GNEP reduces to a standard Nash Equilibrium Problems (NEP), and the Generalized Nash Equilibrium 
reduces to the standard Nash Equilibrium (NE), see [30], [31] and, as an entry point to Game Theory, [32] and [33].

Note that, by definition, at a GNE it is not convenient for players to change their choice unilaterally, that is if the choices made by the other $N-1$ players remain unchanged.

The main existence result for GNEs is probably the one established in [28]. A simplified version given in [34] is here reported for a maximization GNEP:

Theorem 4. Consider a GNEP with $N$ players in which:

1. there exist $N$ non-empty, convex and compact sets $K_{i} \subseteq \mathbb{R}^{n_{i}}$ such that for every $\mathbf{a}=\left(a_{i}, \mathbf{a}_{-i}\right) \in \mathbb{R}^{n}$, where $n:=\sum_{i=1} n_{i}$, with $a_{i} \in K_{i}$ for every $i \in \mathcal{N}, A_{i}\left(\mathbf{a}_{-i}\right) \subseteq K_{i}$;

2. for every $i \in \mathcal{N}, A_{i}(\cdot)$ is a lower semicontinuous and an upper semicontinuous point-to-set map;

3. for every $i \in \mathcal{N}$, the utility function $u_{i}$ is continuous;

4. for every $i \in \mathcal{N}$, the utility function $u_{i}\left(\cdot, \mathbf{a}_{-i}\right)$ is quasi-concave in $a_{i}$ on $A_{i}\left(\mathbf{a}_{-i}\right)$;

therefore a GNE exists.

\section{The Model}

In the present paper I consider a Centralized Dynamic Spectrum Leasing (CDSL) model for power allocation in a frequency band of the radio spectrum. In the frequency band there are two kinds of users: the primary ones, which are the owners of the spectrum rights, and the secondary ones, that have the permission for sharing the band provided they respect some constraints and do not cause harmful interference to the owner of the license.

As already mentioned, in DSL models primary users have an active role in managing the level of secondary interference they are going to tolerate, and they have an incentive, like leasing payments, to allow the secondary users to share their band. While primary users manage their interference cap by varying it, if it is considered to be gainful, the secondary ones manage their transmission powers, in order to obtain a satisfying signal quality without causing an excessive interference.

The model I consider is Centralized (C-DSL) because the multiple primary players participate in the spectrum leasing process under centralized control. 
Suppose there are $M \geq 1$ primary users and $N \geq 1$ secondary users. Say $\mathcal{M}:=\{1, \ldots, M\}$ and $\mathcal{N}:=\{1, \ldots, N\}$ the sets of the indexes for primary and secondary users respectively. The users are pairs of a Transmitter and a Receiver: for primary users, we represent the pairs by $T X_{m}^{p}$ for the transmitter and $R X_{m}^{p}$ for the receiver with $m \in \mathcal{M}$; for secondary users we represent the pairs with a similar notation $T X_{i}^{s}-R X_{i}^{s}$ with $i \in \mathcal{N}$. Consider the set of all the indexes together: $\mathcal{O}:=\{1, \ldots, N, N+1, \ldots N+M\}$ where the first $N$ indexes, from 1 to $N$, represent the secondary users and the last $M$ indexes, from $N+1$ to $N+M$, represent the primary ones. Let $i$ be in $\mathcal{O}$. Note that a signal transmitted by the $i$-th (primary or secondary) transmitter to the $i$-th receiver will create interference at the $j$-th receiver, for every $j \neq i$ primary or secondary. The channel gain between the $i$-th transmitter and $j$-th receiver is denoted by $g_{i, j}$. Say $p_{i}$ the transmission power (in W) of the $i$-th user. The power of the received signal at the $i$-th receiver is therefore $g_{i, i} p_{i}$, while all the other users $j \neq i$ experience at their own receivers, because of the transmission of the $i$-th user, an interference $g_{i, j} p_{i}$.

Therefore, it is clear that for every $m \in \mathcal{M}$ the total secondary interference from all secondary transmission that the $m$-th primary user experiences is given by:

$$
I_{m}:=\sum_{i \in \mathcal{N}} g_{i, m} p_{i} \quad \text { for } m \in \mathcal{M} .
$$

Let us call $I_{0}$ the maximum of these interferences over all the primary users:

$$
I_{0}:=\max _{m \in \mathcal{M}} I_{m}
$$

Another quantity that plays an important role is the Signal to Noise plus Interference Ratio (SINR) $\gamma_{i}$ for the $i$-th secondary user $(i \in \mathcal{N})$ : it is the ratio between the signal (transmitted by the $i$-th secondary transmitter $T X_{i}^{s}$ ) received by the $i$-th secondary receiver $R X_{i}^{s}$ and the total interference plus noise that $R X_{i}^{s}$ receives:

$$
\gamma_{i}:=\frac{g_{i, i} p_{i}}{\sum_{k \in \mathcal{N}, k \neq i} g_{k, i} p_{k}+\sigma_{i}^{2}+\sum_{m \in \mathcal{M}} g_{m, i} p_{m}} \quad \text { for } i \in \mathcal{N}
$$

where $\sigma_{i}^{2}$ (in W) is the Additive White Gaussian Noise (AWGN) at the receiver $R X_{i}^{s}$.

In order to use a simpler notation, put

$$
I_{i}^{r}:=\sum_{k \in \mathcal{N}, k \neq i} g_{k, i} p_{k}+\sigma_{i}^{2}+\sum_{m \in \mathcal{M}} g_{m, i} p_{m} \quad \text { for } i \in \mathcal{N} ;
$$


it represents the total interference plus noise received by the $i$-th secondary user. Therefore, $\gamma_{i}$ defined in (5) can be written as follows:

$$
\gamma_{i}=\frac{g_{i, i} p_{i}}{I_{i}^{r}} \quad \text { for } i \in \mathcal{N}
$$

Say $f\left(\gamma_{i}\right)$ for $i \in \mathcal{N}$ the efficiency function: it represents the probability of correct reception of a signal at a secondary receiver. I assume the typical expression for $f\left(\gamma_{i}\right)$ in the non-coherent FSK (Frequency-Shift Keying) modulation scheme, see [21] for more details. Therefore, it is:

$$
f\left(\gamma_{i}\right)=\left(1-e^{-\frac{1}{2} \gamma_{i}}\right)^{\hat{M}} \quad \text { for } i \in \mathcal{N},
$$

$\hat{M}>0$ being the total number of bits per packet. Therefore, the quantities I need to define the GNEP have been defined, and in the next Section the game will be presented.

\section{The Generalized Game}

In the game, the primary (centralized) system, consisting of multiple primary users, and the secondary users interact by managing the interference cap and the transmission powers, respectively, in order to maximize their own gain. Therefore, we model a game where there is one primary player, the centralized primary system, represented by index 0 , and $N$ secondary players, represented by indexes $i$ with $i \in \mathcal{N}$. For simplicity of notation, let us define a new set for the indexes: say $\mathcal{R}:=\{0\} \cup \mathcal{N}=\{0,1, \ldots, N\}$, where index 0 represents the primary system and the other indexes represent the secondary ones. The non-cooperative GNEP can be defined as follows:

(a) Players are one primary centralized system (consisting of multiple primary users), represented by index 0 , and $N$ secondary users, represented by index $i \in \mathcal{N}$ or equivalently $i \in \mathcal{R}$ with $i \neq 0$.

(b) Exogenous parameter $\omega$ : say $\alpha$ the primary pricing coefficient

$$
\alpha>0
$$

say $\beta$ the maximum pricing value for secondary users, $\delta$ the price weight of the generated interference at the secondary receiver, and $\mu$ the sensitivity 
of the secondary users to interference, see [22]; in the present paper I consider a different range for $\beta, \delta, \mu$ :

$$
\begin{gathered}
0<\delta \leq \beta, \\
0<\mu<\mu^{*}:=\min _{i \in \mathcal{N}}\left\{g_{i i}\left(2 \ln \hat{M} \sum_{k=1, k \neq i}^{N} g_{i, k}\right)\right\} .
\end{gathered}
$$

Players do not have control over the quantities $\varsigma:=-\alpha, \xi:=-\beta, \delta, \zeta:=$ $-\mu$, therefore the vector $\omega:=(\varsigma, \xi, \delta, \zeta) \in \Omega$, where $\Omega:=\{(\varsigma, \xi, \delta, \zeta) \in$ $\left.\mathbb{R}^{4} \mid \varsigma<0, \xi \leq-\delta<0,-\mu^{*}<\zeta<0\right\} \subset \mathbb{R}^{4}$, is an exogenous parameter.

(c) Action space: the primary player, indexed by 0 , makes its decisions on the interference cap $I$, while the secondary players, for $i \in \mathcal{N}$, play the game by managing their transmission powers $p_{i}$. The vector representing the strategy of all the players is $\left(p_{0}, p_{1}, p_{2}, \ldots p_{N}\right) \in A:=A_{0} \times A_{1} \times \ldots \times A_{N}=$ $\prod_{i \in \mathcal{R}} A_{i}$, where $p_{0}=: I$ is the interference cap and belongs to the primary strategy set

$$
A_{0}=\left[0, I_{\max }\right]
$$

where $I_{\max }>0$ represents the maximum interference cap of the primary system (it is fixed in order to ensure a good quality for the primary transmission, because the primary users always first want to achieve a required quality of service), and for $i \in \mathcal{N}, p_{i} \geq 0$ are (non-negative) powers belonging to $A_{i}=A_{i}\left(\mathbf{p}_{-i}\right)$ where

$$
A_{i}\left(\mathbf{p}_{-i}\right):=\left[\tilde{p}_{i, \min }\left(\mathbf{p}_{-i}\right), \tilde{p}_{i, \max }\left(\mathbf{p}_{-i}\right)\right] \quad \text { for } i \in \mathcal{N}
$$

where

$$
\begin{aligned}
& \tilde{p}_{i, \min }\left(\mathbf{p}_{-i}\right):=\min \left\{p_{i, \max }, \frac{2 \ln \hat{M}}{g_{i, i}} I_{i}^{r}\left(\mathbf{p}_{-i}\right)\right\}, \\
& \tilde{p}_{i, \max }\left(\mathbf{p}_{-i}\right):=\min \left\{p_{i, \max }, \frac{I_{i}^{r}\left(\mathbf{p}_{-i}\right)}{\mu \sum_{k=1, k \neq i}^{N} g_{i, k}}\right\},
\end{aligned}
$$

$p_{i, \max }$ being the maximum power level reachable by the secondary $i$-th player. Note that $\frac{2 \ln \hat{M}}{g_{i, i}} I_{i}^{r}\left(\mathbf{p}_{-i}\right)<\frac{I_{i}^{r}\left(\mathbf{p}_{-i}\right)}{\mu \sum_{k=1, k \neq i}^{N} g_{i, k}}$ because of Eq. (11). 
(d) Utility functions: $u_{i}^{\omega}\left(p_{i}, \mathbf{p}_{-i}\right)$ for $i \in \mathcal{R}$ where for the primary system it is

$$
\begin{aligned}
& u_{0}^{\omega}\left(p_{0}, \mathbf{p}_{-0}\right)=u_{0}^{\omega}\left(I, I_{0}\left(\mathbf{p}_{-0}\right)\right) \\
& :=-\left(I-I_{0}\left(\mathbf{p}_{-0}\right)\right)^{2}-\alpha\left(I_{0}\left(\mathbf{p}_{-0}\right)-I\right)\left(\mathrm{e}^{\frac{I_{0}\left(\mathbf{p}_{-0}\right)-I}{I_{0}\left(\mathbf{p}_{-0}\right)}}-1\right)
\end{aligned}
$$

with $I_{0}=I_{0}\left(\mathbf{p}_{-0}\right)$ defined in Eq. (4) and $\alpha$ in Watt, and for the secondary users it is

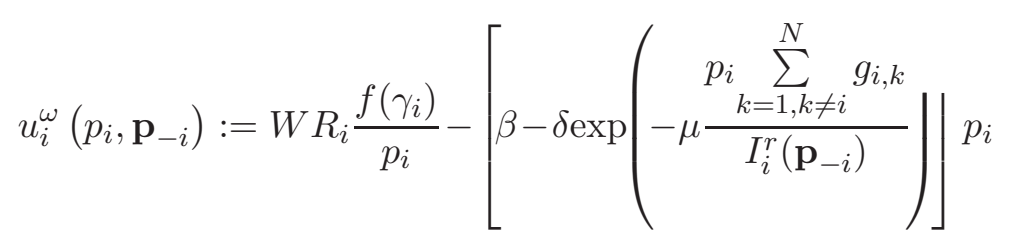

for $i \in \mathcal{N}$

where $W$ is the ratio between the number of information bits per packet and the total number of bits per packet transmitted by the secondary users and $R_{i}$ is the transmission rate of the $i$-th secondary user in bits/sec. The quantity in the square brackets of Eq. (17) is the so-called secondary pricing factor and it is assumed to have units $\frac{\text { bit }}{(\text { Watt })^{2} \times \sec }$ so that the secondary utility function has a consistent unit of measurement.

The primary utility in Eq. (16) is such that the greatest gain for the primary system is obtained when the total interference from the secondary users $I_{0}\left(\mathbf{p}_{-0}\right)$ equals the interference cap $I$. Given $I_{0}\left(\mathbf{p}_{-0}\right)$, the non-positive function of the variable $I$ in Eq. (16) reaches its global maximum when $I=I_{0}\left(\mathbf{p}_{-0}\right)$; when $I>I_{0}\left(\mathbf{p}_{-0}\right)$, the primary system is relatively penalized because useful resources for the secondary players have been wasted; when $I<I_{0}\left(\mathbf{p}_{-0}\right)$, the primary system is significantly penalized because its quality of service target is not reached.

The secondary pricing function in Eq. (17) is the time-continuous version of the one considered in [23], therefore in the present paper the secondary pricing factor cannot be simply considered as a known constant. The secondary utility function is such that the higher the interference generated by a secondary user transmitting at a high power is, the greater the value of pricing it will have to pay is: the presence of the pricing function implicitly induces a sort of cooperation, even if the non-cooperative nature of our game is maintained. On the other hand, the higher the interference $I_{i}^{r}\left(\mathbf{p}_{-i}\right)$ received by the $i$-th secondary user is, the smaller the pricing factor for the $i$-th user is: it implicitly 
involves that if the secondary user $i$ receives a great interference, it is in some way allowed to raise its power without paying an excessive price.

\subsection{Existence of a Generalized Nash Equilibrium}

In this Section, I am going to show that a GNE exists. I follow Theorem 4. Say $K_{i} \subset \mathbb{R}$ the following compact interval for $i \in \mathcal{R}: K_{0}:=\left[0, I_{\max }\right]$ and $K_{i}:=\left[0, p_{i, \max }\right]$ for $i \in \mathcal{N}$; the $N+1$ strategy sets $A_{i}\left(\mathbf{p}_{-i}\right) \subseteq K_{i}$ are nonempty, convex and compact (in the worst case, they are a singleton). For every $i \in \mathcal{N}, A_{i}(\cdot)$ is a lower and upper semicontinuous point-to-set map, because of the continuity of functions $\tilde{p}_{i, \min }\left(\mathbf{p}_{-i}\right)$ and $\tilde{p}_{i, \max }\left(\mathbf{p}_{-i}\right)$. The utility functions $u_{i}^{\omega}$ are trivially continuous for every $i \in \mathcal{R}$. In order to complete the proof of existence af a GNE, one has to show that for every $i \in \mathcal{R}$, the utility function $u_{i}^{\omega}\left(\cdot, \boldsymbol{p}_{-i}\right)$ is quasi-concave in $p_{i}$ on $A_{i}\left(\mathbf{p}_{-i}\right)$. First consider the utility function $u_{0}^{\omega}\left(I, I_{0}\left(\mathbf{p}_{-0}\right)\right)$ for the primary system, see Eq. (16), and derive it with respect to $I$ :

$$
\begin{aligned}
\frac{\partial}{\partial I} u_{0}^{\omega}\left(I, I_{0}\left(\mathbf{p}_{-0}\right)\right) & =2\left(I_{0}\left(\mathbf{p}_{-0}\right)-I\right)+\alpha \mathrm{e}^{\frac{I_{0}\left(\mathbf{p}_{-0}\right)-I}{I_{0}\left(\mathbf{p}_{-0}\right)}} \\
& -\alpha+\alpha \frac{1}{I_{0}\left(\mathbf{p}_{-0}\right)}\left(I_{0}\left(\mathbf{p}_{-0}\right)-I\right) \mathrm{e}^{\frac{I_{0}\left(\mathbf{p}_{-0}\right)-I}{I_{0}\left(\mathbf{p}_{-0}\right)}}
\end{aligned}
$$

with $I_{0}\left(\mathbf{p}_{-0}\right)$ fixed. It results

$$
\frac{\partial}{\partial I} u_{0}^{\omega}\left(I, I_{0}\left(\mathbf{p}_{-0}\right)\right)>0
$$

if and only if

$$
\left(I_{0}\left(\mathbf{p}_{-0}\right)-I\right)\left[2+\frac{\alpha}{I_{0}\left(\mathbf{p}_{-0}\right)} \mathrm{e}^{\frac{I_{0}\left(\mathbf{p}_{-0}\right)-I}{I_{0}\left(\mathbf{p}_{-0}\right)}}\right]>\alpha-\alpha \mathrm{e}^{\frac{I_{0}\left(\mathbf{p}_{-0}\right)-I}{I_{0}\left(\mathbf{p}_{-0}\right)}}
$$

if and only if

$$
I<I_{0}\left(\mathbf{p}_{-0}\right)
$$

and the primary utility function $u_{0}^{\omega}\left(I, I_{0}\left(\mathbf{p}_{-0}\right)\right)$ is quasi-concave in $I$ : its unique local and global maximum on $A_{0}$ is in $I=\min \left\{I_{0}\left(\mathbf{p}_{-0}\right), I_{\max }\right\}$. When it happens that the primary system puts its interference cap equal to $I_{\max }$ and the secondary interference is higher than it, the primary system gives a beacon signal to indicate when the secondary users have absolutely to reduce their transmit powers, see [24]. Otherwise, in order to avoid this, we can set the maximum secondary allowed powers so that the maximum total interference they create (that is $I_{0}$ evaluated in $p_{i, \max }$ for every $i \in \mathcal{N}$ ) is lower than $I_{\max }$. 
The secondary utility functions $u_{i}^{\omega}\left(p_{i}, \mathbf{p}_{-i}\right)$ for $i \in \mathcal{N}$ are quasi-concave for $p_{i} \in A_{i}\left(\mathbf{p}_{-i}\right)$, too. One can compute the first derivative of the secondary utility function $u_{i}^{\omega}$ with respect to $p_{i}$ for every $i \in \mathcal{N}$, for $\mathbf{p}_{-i}$ fixed:

$$
\begin{aligned}
\frac{\partial u_{i}^{\omega}}{\partial p_{i}}\left(p_{i}, \mathbf{p}_{-i}\right) & =\frac{W R_{i}}{p_{i}^{2}}\left[f^{\prime}\left(\gamma_{i}\right) \gamma_{i}-f\left(\gamma_{i}\right)\right] \\
& -\left[\beta-\delta \exp \left(\begin{array}{c}
p_{i} \sum_{k=1, k \neq i}^{N} g_{i, k} \\
I_{i}^{r}\left(\mathbf{p}_{-i}\right)
\end{array}\right)\right] \\
& -\left[\delta \mu \frac{\sum_{k=1, k \neq i}^{N} g_{i, k}}{I_{i}^{r}\left(\mathbf{p}_{-i}\right)} \exp \left(-\mu \frac{p_{i} \sum_{k=1, k \neq i}^{N} g_{i, k}}{I_{i}^{r}\left(\mathbf{p}_{-i}\right)}\right)\right] p_{i} .
\end{aligned}
$$

By equaling the right hand side of Eq. (22) to zero, we put a necessary condition for $p_{i}$ to be a local maximum point for $u_{i}^{\omega}\left(p_{i}, \mathbf{p}_{-i}\right)$ for a fixed vector $\mathbf{p}_{-i}$. By taking into consideration Eq. (7), one obtains for every $i \in \mathcal{N}$ :

$$
\begin{aligned}
& f^{\prime}\left(\frac{g_{i, i} p_{i}}{I_{i}^{r}\left(\mathbf{p}_{-i}\right)}\right) \frac{g_{i, i} p_{i}}{I_{i}^{r}\left(\mathbf{p}_{-i}\right)}-f\left(\frac{g_{i, i} p_{i}}{I_{i}^{r}\left(\mathbf{p}_{-i}\right)}\right) \\
& =\frac{1}{W R_{i}}\left\{\left[\beta-\delta \exp \left(-\mu \frac{p_{i} \sum_{k=1, k \neq i}^{N} g_{i, k}}{I_{i}^{r}\left(\mathbf{p}_{-i}\right)}\right\rfloor\right\} p_{i}^{2}\right. \\
& +\frac{1}{W R_{i}}\left\{\left[\delta \mu \frac{\sum_{k=1, k \neq i}^{N} g_{i, k}}{I_{i}^{r}\left(\mathbf{p}_{-i}\right)} \exp \left(-\mu \frac{p_{i} \sum_{k=1, k \neq i}^{N} g_{i, k}}{I_{i}^{r}\left(\mathbf{p}_{-i}\right)}\right) \mid\right\} p_{i}^{3} .\right.
\end{aligned}
$$

Note that if Eq. (23) holds, each of its solutions $p_{i}$ is a candidate to be a local maximum point of $u_{i}^{\omega}$ for $\mathbf{p}_{-i}$ fixed. It is not possible to find an explicit solution, anyway we can solve the transcendental equation (23) by a graphical method. Say

$$
\operatorname{LHS}\left(p_{i}, I_{i}^{r}\left(\mathbf{p}_{-i}\right)\right):=f^{\prime}\left(\frac{g_{i, i} p_{i}}{I_{i}^{r}\left(\mathbf{p}_{-i}\right)}\right) \frac{g_{i, i} p_{i}}{I_{i}^{r}\left(\mathbf{p}_{-i}\right)}-f\left(\frac{g_{i, i} p_{i}}{I_{i}^{r}\left(\mathbf{p}_{-i}\right)}\right)
$$




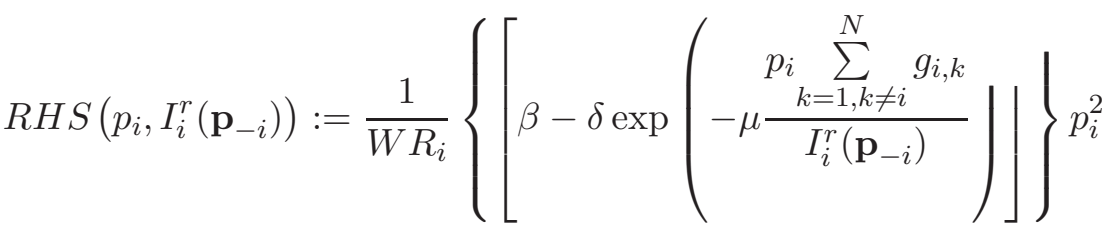

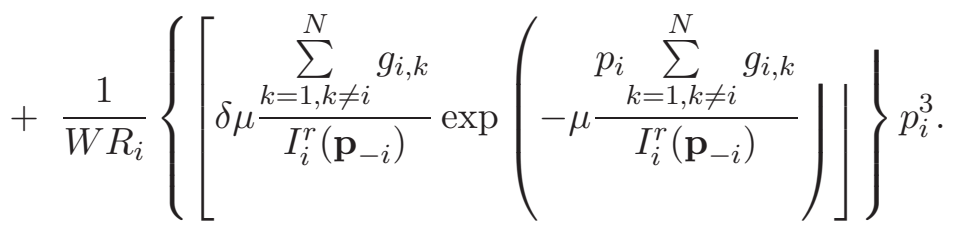

Function $\operatorname{LHS}\left(p_{i}, I_{i}^{r}\left(\mathbf{p}_{-i}\right)\right)$ shows the following behavior: it is positive and monotonically increasing for $p_{i} \in\left(0, \frac{2 \ln \hat{M}}{g_{i, i}} I_{i}^{r}\left(\mathbf{p}_{-i}\right)\right)$, reaches its local and global maximum for $p_{i}=\frac{2 \ln \hat{M}}{g_{i, i}} I_{i}^{r}\left(\mathbf{p}_{-i}\right)$ and monotonically decreases for

$$
p_{i}>\frac{2 \ln \hat{M}}{g_{i, i}} I_{i}^{r}\left(\mathbf{p}_{-i}\right)
$$

tending to -1 when $p_{i}$ tends to $+\infty$. Therefore, $\operatorname{LHS}\left(p_{i}, I_{i}^{r}\left(\mathbf{p}_{-i}\right)\right)$ is monotonically decreasing in the domain $A_{i}\left(\mathbf{p}_{-i}\right)$. Function $R H S\left(p_{i}, I_{i}^{r}\left(\mathbf{p}_{-i}\right)\right)$ is strictly positive and monotonically increasing in $A_{i}\left(\mathbf{p}_{-i}\right)$, see conditions (10) and (11). The graphic of $R H S\left(p_{i}, I_{i}^{r}\left(\mathbf{p}_{-i}\right)\right)$ for $p_{i} \in A_{i}\left(\mathbf{p}_{-i}\right)$ is placed in the first quadrant between the graphics of the two cubic functions $y_{1}\left(p_{i}, I_{i}^{r}\left(\mathbf{p}_{-i}\right)\right):=$ $(\beta-\delta) p_{i}^{2}+\delta a \exp (-1) p_{i}^{3}$ and $y_{2}\left(p_{i}, I_{i}^{r}\left(\mathbf{p}_{-i}\right)\right):=(\beta-\delta \exp (-1)) p_{i}^{2}+\delta a p_{i}^{3}$, where we put $a:=\frac{\mu \sum_{k=1, k \neq i}^{N} g_{i, k}}{I_{i}^{r}\left(\mathbf{p}_{-i}\right)}$. By resuming the preceding results, we can state that for $p_{i} \in A_{i}\left(\mathbf{p}_{-i}\right)$ and $I_{i}^{r}\left(\mathbf{p}_{-i}\right)$ fixed, function $\operatorname{LHS}\left(p_{i}, I_{i}^{r}\left(\mathbf{p}_{-i}\right)\right)$ is monotonically decreasing, starting from its positive maximum $L H S\left(\frac{2 \ln \hat{M}}{g_{i, i}} I_{i}^{r}\left(\mathbf{p}_{-i}\right), I_{i}^{r}\left(\mathbf{p}_{-i}\right)\right)$, while $\operatorname{RHS}\left(p_{i}, I_{i}^{r}\left(\mathbf{p}_{-i}\right)\right)$ is positive and monotonically increasing. Three cases are possible: the first one is $\operatorname{LHS}\left(p_{i}, I_{i}^{r}\left(\mathbf{p}_{-i}\right)\right)>R H S\left(p_{i}, I_{i}^{r}\left(\mathbf{p}_{-i}\right)\right.$ for every $p_{i} \in A_{i}\left(\mathbf{p}_{-i}\right)$, and it implies that function $u_{i}^{\omega}$ is strictly increasing (and the unique global maximum point is $\left.p_{i}=\tilde{p}_{i, \max }\right)$; the second case is $\operatorname{LHS}\left(p_{i}, I_{i}^{r}\left(\mathbf{p}_{-i}\right)\right)<R H S\left(p_{i}, I_{i}^{r}\left(\mathbf{p}_{-i}\right)\right)$ for every $p_{i} \in A_{i}\left(\mathbf{p}_{-i}\right)$, that is $u_{i}^{\omega}$ strictly decreasing (and the unique global maximum point is $p_{i}=\frac{2 \ln \hat{M}}{g_{i, i}} I_{i}^{r}\left(\mathbf{p}_{-i}\right)$ ); the third case is $\operatorname{LHS}\left(p_{i}, I_{i}^{r}\left(\mathbf{p}_{-i}\right)\right)=R H S\left(p_{i}, I_{i}^{r}\left(\mathbf{p}_{-i}\right)\right)$ for one (and only one) $p_{i} \in A_{i}\left(\mathbf{p}_{-i}\right)$. It is easy to verify that such a power value is a local maximum point and that it is the unique global maximum point. Note that maybe other intersections occur between the graphs of $L H S$ and $R H S$ for $p_{i} \notin A_{i}\left(\mathbf{p}_{-i}\right)$, but 
if there is one intersection in $A_{i}\left(\mathbf{p}_{-i}\right)$, then it is unique in $A_{i}\left(\mathbf{p}_{-i}\right)$. We can conclude that for every $i \in \mathcal{N}$ the secondary utility $u_{i}\left(\cdot, \mathbf{p}_{-i}\right)$ is quasi-concave in $p_{i}$ on $A_{i}\left(\mathbf{p}_{-i}\right)$.

Therefore, the assumptions of Theorem 4 are fulfilled and a GNE exists.

\section{Conclusions}

I have proposed a game is in a C-DSL context, where primary users, under a centralized control, have an active role in managing the level of secondary interference they are going to tolerate, and they have an incentive, like leasing payments, to allow the secondary users to share their band. The secondary users play the game by managing their own transmission power. The mathematical treatment is dedicated to the proof of existence of a Generalized Nash Equilibrium to which the proposed Generalized Nash Equilibrium Problem converges. The main difficulty in treating Generalized Nash Equilibrium Problems is that the strategy sets of the mobiles are not independent of each other. By choosing a suitable set of actions for every player, letting the primary players manage the tolerated secondary interference and the secondary players manage their own transmission powers, I have proven the existence of a Nash Equilibrium for the game. The request I have made on the strategy sets of secondary players is very natural, because it just implies that the secondary SINR is bounded from below and above.

\section{References}

[1] Federal Communications Commission (FCC) - Spectrum policy task force, Report, ET Docket No. 02-135 (November 2002) [online]. Available from: http://hraunfoss.fcc.gov/edocs_public/attachmatch/DOC228542A1.pdf [Accessed January 7, 2013].

[2] Federal Communications Commission (FCC), Notice of proposed rule making and order, ET Docket No 03-322 (December 2003) [online]. Available from: http://hraunfoss.fcc.gov/edocs_public/attachmatch/FCC-03322A1.pdf [Accessed January 7, 2013].

[3] Committee on Scientific Use of the Radio Spectrum; Committee on Radio Frequencies; Board on Physics and Astronomy; Division on Engineering and Physical Sciences; National Research Council. Spectrum Management 
for Science in the 21st Century, The National Academies Press, Washington, D.C. (2010).

[4] F.K. Jondral, From Maxwell's Equations to Cognitive Radio, Proceedings of the 3rd International Conference on Cognitive Radio Oriented Wireless Networks and Communications, CrownCom 2008, 15-17 May 2008, Singapore (2008).

[5] Radio Spectrum Policy Group (RSPG), RSPG Opinion on the Radio Spectrum Policy Programme, RSPG10330 Final (June 2010) [online]. Available from: http://rspg.groups.eu.int/_documents/documents/opinions/rspg10_330_rspp_opinion.pdf [Accessed January 7, 2013].

[6] Radio Spectrum Policy Group (RSPG) Report on Collective use of Spectrum (CUS) and other spectrum sharing approaches, RSPG11-392 Final (November 2011) [online]. Available from: http://rspg.groups.eu.int/_documents/documents/meeting/rspg26/rspg11_392_report_CUS_other_approaches_final.pdf [Accessed January 7, 2013].

[7] B. Gavish, An improved method for auctioning wireless spectrum, International Journal of Pure and Applied Mathematics, 7, Issue 2 (2003), 175206.

[8] G. Li, G.L. Stüber, Orthogonal Frequency Division Multiplexing for Wireless Communications, New York: Springer (2006).

[9] F. Tamburini, E. Mari, A. Sponselli, B. Thidé, A. Bianchini, F. Romanato, Encoding many channels on the same frequency through radio vorticity: first experimental test. New Journal of Physics, 14033001 (2012), 1-17.

[10] J.III Mitola, G.Q.Jr Maguire, Cognitive radio: making software radios more personal, IEEE Personal Communications, 6, Issue 4 (1999), 13-18.

[11] J.III Mitola, Cognitive radio: An integrated agent architecture for software defined radio, Dissertation for Doctor of Technology, Royal Inst. Technol. (KTH), ISSN 1403-5286, ISRN KTH/IT/AVH 00/01,-SE, Stockholm, Sweden (2000).

[12] S. Haykin, D.J. Thomson, J.H. Reed, Spectrum sensing for cognitive radio, Proceedings of the IEEE, 97, (2009), 849-877. 
[13] R. Tandra, S.M. Mishra, A. Sahai, What is a spectrum hole and what does it take to recognize one?, Proceedings of the IEEE, 97 (2009), 824-848.

[14] I.F. Akyildiz, W.Y. Lee, M.C. Vuran, S. Mohanty, NeXt generation/dynamic spectrum access/cognitive radio wireless networks: A survey, Computer Networks, 50 (2006), 2127-59.

[15] B. Wang, Y. Wu, K.J.R. Li, Game Theory for Cognitive Radio Networks: an Overview, Computer Networks, 54, Issue 14 (October 2010), 2537-61.

[16] D. Famolari, N. Mandayam, D. Goodman, V. Shah, A new framework for power control in wireless data networks: games, utility, and pricing, In: Wireless Multimedia Network Technologies, eds. Ganesh, Pahlavan, and Zvonar, Kluwer Academic (1999), 289-310.

[17] N. Feng, N.B. Mandayam, D.J. Goodman, Joint power and rate optimization for wireless data services based on utility functions, Proc. CISS, 1 (1999), 109-113.

[18] D. Goodman, N. Mandayam, Power control for wireless data, IEEE Pers. Comm. Mag., 7 (2000), 45-54.

[19] H. Ji, D. Huang, Non-cooperative uplink power control in cellular radio systems, Journal Wireless Networks - Special issue transmitter power control, 4, Issue 3 (March 1998), 233-240.

[20] V. Shah, N. Mandayam, D. Goodman, Power Control for Wireless Data based on Utility and Pricing, Proc. PIMRC '98 (1998), 1427-32.

[21] C.U. Saraydar, N. Mandayam, D. Goodman, Efficient power control via pricing in wireless data networks, IEEE Trans. Comm., 50 (2002), 291303.

[22] C.K. Tan, M.L. Sim, T.C. Chuah, Fair power control for wireless ad hoc networks using game theory with pricing scheme, IET Commun., 4, Issue 3 (2010), 322-333.

[23] E. Del Re, R. Pucci, L.S. Ronga, Energy Efficient Resource Allocation Game for Cognitive Radio, CogART '11: Proceedings of the 4th International Conference on Cognitive Radio and Advanced Spectrum Management, Barcelona, Spain, October 26-29, (2011). 
[24] S.K. Jayaweera, T. Li, Dynamic Spectrum Leasing in Cognitive radio Networks via Primary-Secondary User Power Control Games, IEEE Transactions On Wireless Communications, 8, Issue 6 (2009), 3300-10.

[25] G. Vasquez-Vilar, C. Mosquera, S.K. Jayaweera, Primary Users enter the Game: Performance of Dynamic Spectrum Leasing in Cognitive Radio Networks, IEEE Transactions On Wireless Communications, 9, Issue 12 (2010), 3625-29.

[26] K. Hakim, S.K. Jayaweera, G. El-howayek, C. Mosquera, Efficient Dynamic Spectrum Sharing in Cognitive Radio Networks: Centralized Dynamic Spectrum Leasing (C-DSL), IEEE Transactions On Wireless Communications, 9 (2010), 2956-66.

[27] G. Debreu, A social equilibrium existence theorem, Proceedings of the $\mathrm{Na}$ tional Academy of Sciences of the USA, 38 (1952), 886-893.

[28] K.J. Arrow, G. Debreu, Existence of an equilibrium for a competitive economy, Econometrica, 22 (1954), 265-290.

[29] F. Facchinei, C. Kanzow, Generalized Nash Equilibrium Problems, Ann. Oper. Res., 175 (2010), 177-211.

[30] J.F. Nash, Equilibrium Points in n-Person Games, Proc. Nat. Acad. Sci. U.S.A., 36 (1950), 48-49.

[31] J.F. Nash, Non-cooperative Games, Ann. of Math., 54 (1951), 286-295.

[32] J. von Neumann, O. Morgenstern, Theory of Games and Economic Behavior, Princeton University Press (1944); Second Edition (1947).

[33] D. Fudenberg, J. Tirole, Game Theory, Cambridge, MA: MIT Press (1991).

[34] T. Ichiishi, Game Theory for Economic Analysis, New York: Academic press (1983). 